Research Article

\title{
Signal Nonstationary Degree Evaluation Method Based on Moving Statistics Theory
}

\author{
Haoxiang He $\mathbb{D}^{1}{ }^{1}$ Shitao Cheng, ${ }^{1}$ and Xiaofu Zhang ${ }^{2}$ \\ ${ }^{1}$ Beijing Key Lab of Earthquake Engineering and Structural Retrofit, Beijing University of Technology, Beijing 100124, China \\ ${ }^{2}$ China Academy of Building Research, Beijing 100013, China \\ Correspondence should be addressed to Haoxiang He; hhx7856@163.com
}

Received 26 February 2021; Revised 16 April 2021; Accepted 27 April 2021; Published 4 May 2021

Academic Editor: Antonio Batista

Copyright (C) 2021 Haoxiang He et al. This is an open access article distributed under the Creative Commons Attribution License, which permits unrestricted use, distribution, and reproduction in any medium, provided the original work is properly cited.

Nonstationary signal refers to the signal whose statistics change with time, and its nonstationary degree evaluation can provide effective support for the evaluation of the operating state of the signal source. This paper introduced a variety of typical signal global and local nonstationary degree evaluation methods and compared the applicable scope of different evaluation methods. In view of the limitations of the existing evaluation methods in the scope of application, considering the influence of adjacent signal points, this paper proposed the concepts and calculation methods of the moving mean, moving standard deviation, moving variation coefficient, and moving Hurst exponent based on the theory of moving statistics. According to different nonstationary degree evaluation methods, three different fields of signals (sinusoidal signal, mechanical fault signal, and ECG signal) are analyzed. The results show that, compared with the existing nonstationary degree evaluation methods, the signal nonstationary degree evaluation method proposed in this paper can reveal the time-varying details of the nonstationary signals, with high precision and strong stability, and has unique advantages in nonstationary signal processing.

\section{Introduction}

A large number of measured data and related studies show that the signals obtained from nature and engineering applications are usually random or stochastic due to their own characteristics and noise effects. Since the random signal can neither be described by deterministic time function nor be reproduced accurately, it must be studied by the statistical method. In different fields of science and engineering, researchers need to obtain physical parameters or statistics that can represent time-varying random signals according to their individual needs and then carry out signal identification or diagnosis. Therefore, it is significant to extract statistical features that can fully represent the signal random characteristics.

The usual random signal statistics include mean value (first-order statistics), variance and correlation function (second-order statistics), and higher-order statistics such as third-order and fourth-order or higher-order moments, higher-order cumulants, and higher-order spectrum. If the change of the statistic is time independent, the signal is called stationary signal. If the statistic changes with time, the signal is called nonstationary signal [1-3]. The random signal in engineering is usually nonstationary signal. When the stationary signal is mixed with nonstationary noise, the nonstationary part can be removed by filtering or smoothing technology, so as to retain the actual information of the signal. When the nonstationary degree of the signal can represent the physical or essential characteristics of the signal source, it is necessary to carry out intensive research on the time-frequency variation characteristics and extract significant information to provide technical support for feature recognition and spectrum analysis $[4,5]$. When the nonstationarity of the signal can represent the short-term or long-term variation of the signal, it also needs to pay attention to its time-varying characteristics, a local distortion degree, trend term, and envelope characteristics. These features can provide a basis for signal classification and fault diagnosis [6]. Thus, it is very important to study the degree of signal nonstationarity [7]. 
With the further understanding of nonstationary signals, many assumptions of stationary signals in engineering applications are not entirely correct. The analysis and processing of nonstationary signals have grown up to be an important issue in modern signal processing.

In terms of nonstationary signal analysis, according to the different technical means adopted, it can be roughly divided into two categories: nonparametric method and parametric method. In addition, according to the different analysis domain of each method, the above two categories are divided into time-domain methods, frequency domain methods, and time-frequency-domain methods [8-10], respectively.

The nonparametric method processes the signal directly through various transformations. The time-domain methods of nonparametric methods mainly include total energy, cumulative energy, Teager energy operator (TEO), time moment of energy, power, signal envelope, and average time. The frequency domain methods in the nonparametric method mainly include Fourier spectrum, classical spectral estimation method of power spectrum, high-order spectral analysis, average frequency, spectral moment, and other spectral parameters [11]. The time-frequency-domain nonparametric methods mainly include wavelet decomposition, Wigner-Ville distribution (WVD) decomposition, and Hilbert-Huang transform (HHT) method [12]. Although the domain decomposition signals can be obtained by the time-frequency analysis method, but due to the influence of frequency band aliasing or cross term interference, its rigor, accuracy, and versatility need to be further improved.

Parametric method is also called model-based method. Firstly, parametric model is established according to the signal [13], and then the signal is analyzed and processed according to the model parameters. Among them, the timedomain method is mainly based on the ARMA model [14] and the AR model [15] of modern spectrum estimation method; the frequency domain method is mainly based on the signal power spectrum parametric modeling method; the time-frequency-domain method is mainly based on the time-varying ARMA model and the time-varying basis function ARMA model of instantaneous spectrum estimation method.

It should be pointed out that statistics, transformation, decomposition, and other processing on the signal are usually used in the above methods. The purpose is to extract the time-frequency-domain information and details of the signal. However, the quantification of nonstationary signal, i.e., nonstationary degree evaluation, can reveal the signal characteristics from the essence of the signal and identify the intrinsic property, which is also necessary for many engineering applications.

Although the definitions of signal stationarity and nonstationarity are relatively clear, the actual process of signal nonstationary degree evaluation is relatively complex and there is a lack of generally applicable quantitative index. Due to the limitations of theoretical development, many nonstationary signal processing methods are relatively simplified or have their own shortcomings; both qualitative analysis and quantitative analysis are not comprehensive and systematic, so intensive research is necessary.

In view of this, this paper introduced a variety of signal nonstationary degree evaluation methods and compared the applicable scope and limitations of these methods. Considering the time-varying characteristics of nonstationary signals, several signal nonstationary degree evaluation methods such as moving variation coefficient are proposed based on moving statistical characteristics. The analysis of different types of signals proves that the methods proposed in this paper have good recognition ability and can accurately characterize the nonstationary degree of nonstationary signal. The research block diagram of this paper is shown in Figure 1.

\section{Nonstationary Degree Evaluation Method}

The evaluation and analysis of nonstationary signals are an urgent problem to be discussed in many research fields. There are various methods in different fields to judge whether a given signal has nonstationarity or not and to evaluate the extent of nonstationarity, i.e., nonstationary degree. For example, unit root test method [16-18] and average entropy theory $[19,20]$ are often used in econometrics. Recurrence plot [21-24] is usually used in nonlinear system analysis. Hurst exponent is used in stock analysis [25]. Crossing-zero rate [26] and nonlinear cross prediction method [27] are used in seismic signal analysis. The advantages and disadvantages of the above methods are different, and appropriate methods should be selected according to the engineering requirements and characteristics in practical application. Generally speaking, the above methods can be divided into the global evaluation method and the local evaluation method. The former focuses on the whole nonstationary degree of the signal and only uses a single value for evaluation. The latter can appropriately reveal the nonstationary degree of the local or details of the signal, and its value is a set of vectors. In order to select a suitable nonstationary degree evaluation method according to the signal characteristic requirements, this study introduces, analyses, and discusses the application scope and characteristics of typical algorithms.

\subsection{Evaluation Method of Signal Global Nonstationary Degree.} The global nonstationary degree evaluation method of signals is to determine the nonstationary degree of the signal based on corresponding indicators. The method for judging the global nonstationary degree of signals is simple to operate and can directly and qualitatively represent the nonstationary degree of signals. Evaluation methods of signal global nonstationary degree mainly include autocorrelation function method, unit root test, information entropy method, and recurrence plot method.

Autocorrelation function can describe the correlation between adjacent variables of time series and express the degree of correlation at different times of the same process. If the autocorrelation function of a random time series signal decreases rapidly to zero with the increase of delay, it can be 


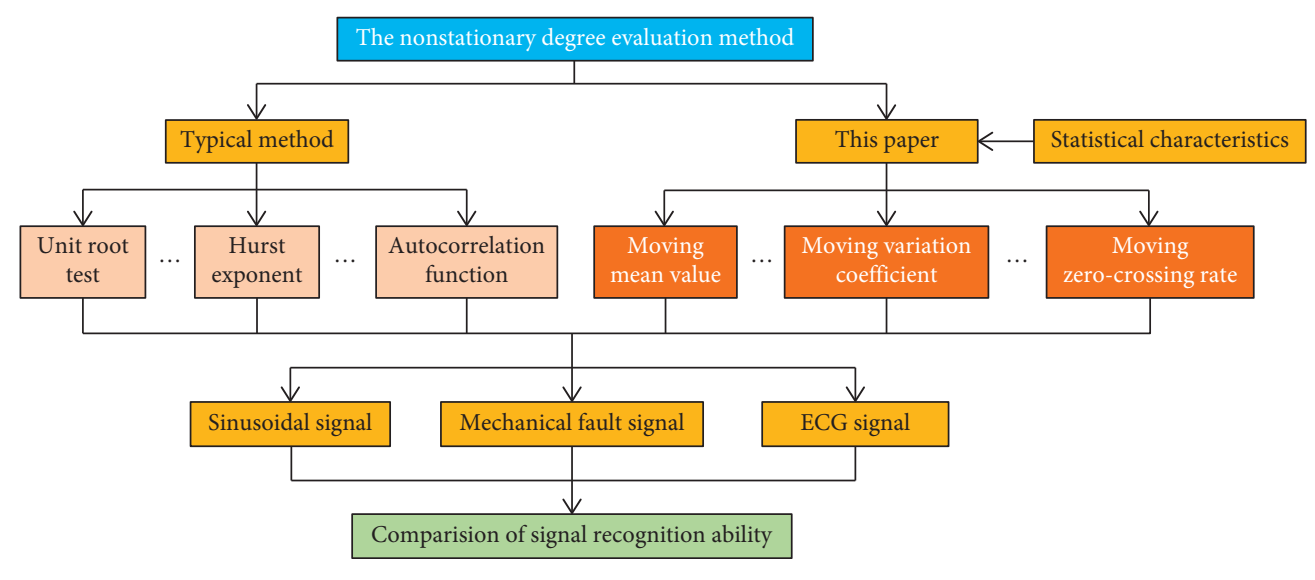

FIGURE 1: Block diagram of this paper research.

considered as a stationary sequence. If the autocorrelation function does not decrease rapidly to zero with the increase of delay, it can be considered as a nonstationary sequence. The autocorrelation function method is simple and intuitive but due to the actual signal it has finite duration and is bounded, and with the increase of delay, the dimension of data that can participate in the calculation will decrease. At this time, the autocorrelation function has been difficult to fully and accurately evaluate the stability degree of the signal in the later period.

The unit root test refers to determining the nonstationary degree of the sequence by checking whether there is a unit root in the sequence [16]. If there is a unit root in the sequence, the signal is not stable. There are many methods for unit root test, including ADF test, PP test, and NP test. Because the unit root test is a kind of qualitative judgment for the evaluation of signal nonstationarity, but not quantitative analysis, it can only judge whether the signal is stable or nonstationary according to whether $\rho$ is equal to 1 , so it cannot be used to quantitatively evaluate the nonstationary degree of different signals [28].

The nonstationary degree based on information entropy is to evaluate the nonstationarity of the signal by extracting the upper bound of the information entropy in the stable information structure of the original signal [29]. When the value approaches 0 , the signal is considered to be nonstationary; when the value approaches 1 , the signal is considered to be stationary, but the distinction between stationary and nonstationary is not clear enough. In addition, for different types of signals, the upper bound of information entropy has little difference, so it is impossible to use information entropy to compare the nonstationary degree of different signals. This leads to the limitation of the application and extension of information entropy [30].

Recurrence plot analysis is a signal processing method that uses the principle of phase space reconstruction and shows the recursive characteristics of the system. It mainly measures the nonstationary degree of time series through the periodic trajectory of phase space visualization [31]. The recurrence plot is very sensitive to the change of system trajectory to time in the state space. This method can directly analyze the trajectory in the high-dimensional space.
However, the evaluation ability of this method is still limited for some long-term signals with strong nonstationarity and the image representation is not fully applicable in some fields.

2.2. Evaluation Method of Local Nonstationary Degree of Signals. Although the global nonstationary degree evaluation method can qualitatively evaluate the nonstationary degree of the signal, it cannot reveal the time-varying characteristics of the signal and cannot meet the precision requirements. Some random signals are stationary on the whole, but contain the characteristics of nonstationarity and mutation in local segment. In order to judge the stationary change of signal comprehensively and intensively, it is necessary to observe the whole fluctuation and local mutation of signal simultaneously. The local nonstationary degree evaluation method can be used to calculate the statistic change quantitatively. Typical methods for evaluating local nonstationary degree of signals mainly include nonlinear cross prediction method, stationary degree method based on Hilbert-Huang Transform (HHT), crossing-zero rate and Hurst graph method.

Based on the nonlinear cross prediction method, the signal is divided into several adjacent segments, and the cross prediction errors between segments are used to characterize the degree of difference and stability of the time series of each segment. The advantage of this method is that the nonstationary degree calculation takes into account not only periodic fluctuations, but also the time series trend changes with time, so it is easy to obtain more stable calculation results. However, this method can only be used as an auxiliary method because it is sensitive to the amplitude and lacks of clear judgment threshold.

Crossing-zero rate represents the number of times a signal passes through zero points in a unit time. It can be used to indicate the characteristics of the frequency or period of the signal changing with time, so it can show the frequency of nonstationary degree in a short time. However, if the high-frequency part of the signal is prominent and does not intersect with the zero line completely, it is difficult to identify by the crossing-zero rate, which is prone to high- 
frequency omission. Therefore, the zero-crossing rate is not accurate enough to express the nonstationary degree of the signal. Generally, it has to be combined with other methods to make a comprehensive evaluation.

Hurst exponent is a kind of indicator to judge whether time series data comply with random walk or biased random walk process, which is calculated by the rescaled range $(R / S)$ analysis method [32, 33]. In addition, Hurst exponent can also measure the change of signal trend strength and noise level with time. Therefore, it can be used as the evaluation basis of signal nonstationary degree. To calculate Hurst exponent, the cumulative deviation $x_{i, a}$ of the signal should be obtained according to the following formula:

$$
x_{i, a}=\sum_{u-1}^{i}\left(x_{u, a}-m_{a}\right), \quad i=1,2, \ldots, n,
$$

where $m_{a}$ is the mean value of $x_{u, a}$ in the $a^{\text {th }}$ interval and $x_{u, a}$ is the cumulative deviation of $t^{\text {th }}$ element in $a^{\text {th }}$ interval.

The range $R_{a}$ is defined as the difference between the maximum and minimum of the accumulated deviation $x_{t, a}$. If $S_{a}$ is used to indicate the sample standard deviation of $a^{\text {th }}$ subinterval, the rescaling range $R_{a} / S_{a}$ can be determined, and the mean of all the rescaling ranges of $A$ is as follows:

$$
(R / S)_{l}=\frac{1}{A} \sum_{a-1}^{A}\left(R_{a} / S_{a}\right) .
$$

If the subinterval length $l$ is variable, then (2) can be expressed as follows:

$$
(R / S)_{l}=K l^{H}
$$

where $K$ is a constant and $H$ is the Hurst exponent. Take the logarithm about the two sides of (3), given by

$$
\log \left((R / S)_{l}\right)=\log (K)+H \log (l) .
$$

It can be seen that the Hurst exponent obtained by $R / S$ analysis of nonlinear time series can effectively predict time series. It represents the degree of self-similarity of data and its second-order statistics and can be used to judge the nonstationarity of stochastic processes.

Based on the characteristics of the signal itself, the Hilbert-Huang transform (HHT) uses the empirical mode decomposition (EMD) method to decompose the signal into a series of intrinsic mode functions and then performs the Hilbert transform on the intrinsic mode function components to obtain the energy distribution in the time-frequency domain, which is called Hilbert spectrum $H(\omega, t)$ [34-37]. On this basis, Huang proposed the concept of energy spectrum $I E(t)$ and frequency domain nonstationary degree $D_{s}(t)$ to reveal the distribution variation of the signal in the frequency domain. The specific calculation process is as follows.

Hilbert marginal spectrum $h(\omega)$ can be obtained by integrating $H(\omega, t)$ on time $t$, which is used to represent the distribution of signal energy on frequency $\omega$. The corresponding formula is

$$
h(\omega)=\int_{0}^{T} H(\omega, t) \mathrm{d} t .
$$

On this basis, the average marginal spectrum $n(\omega)$ can be calculated according to the following formula:

$$
n(\omega)=\frac{1}{T} h(\omega)
$$

Then the frequency domain nonstationary degree $D_{s}(\omega)$ can be expressed as

$$
D_{s}(\omega)=\frac{1}{T} \int_{0}^{T}\left[1-\frac{H(\omega, t)}{n(\omega)}\right]^{2} \mathrm{~d} t .
$$

Similar to the definition of (7), stationary degree in time domain can be defined as a function of time. Therefore, Hilbert energy spectrum $I E(t)$ can be obtained by integrating $H(\omega, t)$ in frequency domain, which indicates the change of signal energy with time, and the expression is put forward in this study as follows:

$$
I E(t)=\int_{0}^{\omega_{\max }} H(\omega, t) \mathrm{d} \omega .
$$

The formula for calculating the average energy spectrum $n(t)$ is as follows:

$$
n(t)=\frac{1}{\omega_{\max }} I E(t) .
$$

Therefore, the time-domain stationary degree $D_{s}(t)$ of the signal can be expressed as

$$
D_{s}(t)=\frac{1}{\omega_{\max }} \int_{0}^{\omega_{\max }}\left[1-\frac{H(\omega, t)}{I E(t)}\right]^{2} \mathrm{~d} \omega .
$$

Although HHT method can directly represent stationarity by using three-dimensional distribution about amplitude-frequency-time, there are still many limitations in theory; especially EMD decomposition has no clear mathematical mechanism, and the realization of signal decomposition stability and completeness lacks a theoretical basis. Therefore, the analysis of HHT-based nonstationary degree needs to be further studied and promoted.

In summary, the characteristics and deficiencies of the above various nonstationary degree evaluation methods are reviewed and compared, and the results are shown in Table 1. It can be seen that each method has its own characteristics, but at the same time there are many limitations and disadvantages, which need to be further studied and improved.

\section{Nonstationary Degree Evaluation Method Based on Moving Statistics}

Although the actual signals in various engineering fields show stochastic characteristics, due to the influence of the generation mechanism and noise characteristics, each point in the signal is related to the nearby points. For most signals, it is usually the adjacent signal that has a greater impact on the signal point itself, while the signal far away has a minor impact on the center point. The nonstationary degree of random signals can be evaluated more comprehensively by 
TABLE 1: Comparison of the applicable scope of the nonstationary degree evaluation method.

\begin{tabular}{|c|c|c|c|c|c|}
\hline \multicolumn{6}{|l|}{ Characteristic } \\
\hline Method & Theoretical basis & Integrality & Locality & Time domain & Frequency domain \\
\hline Autocorrelation function & $\bullet$ & 0 & $\bullet$ & $\bullet$ & 0 \\
\hline Unit root test & - & ○ & $\mathrm{O}$ & ○ & $\mathrm{O}$ \\
\hline Information entropy method & $\mathrm{O}$ & ○ & $\mathrm{O}$ & ○ & $\mathrm{O}$ \\
\hline Recurrence plot & ○ & ○ & $\bullet$ & ○ & $\mathrm{O}$ \\
\hline Nonlinear cross prediction method & 0 & 0 & 0 & 0 & 0 \\
\hline Crossing-zero rate & $\mathrm{O}$ & ○ & $\bullet$ & $\bullet$ & ○ \\
\hline Hurst exponent & 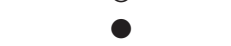 & 0 & 0 & 0 & 0 \\
\hline HHT nonstationary degree & O & ○ & - & - & - \\
\hline Moving zero-crossing rate & 0 & 0 & $\bullet$ & $\bullet$ & 0 \\
\hline Moving Hurst exponent & - & - & - & - & O \\
\hline Moving statistics & $\bullet$ & - & $\bullet$ & 0 & - \\
\hline
\end{tabular}

- indicates that it has the corresponding performance and $\mathrm{O}$ indicates that it does not have the corresponding performance.

considering the relationship among different signal points and their interaction ability.

In order to underline the above characteristics, the weighted moving statistics method can usually be used to calculate each point in the signal. In other words, data smoothing can be achieved by assigning weights to the signal points in the specified range and replacing the points with statistics in the corresponding range, so as to obtain more effective signal characteristics. Using weighted moving statistics method to process signals is equivalent to low-pass filtering, and the corresponding filters have the characteristics of smooth transition from passband to stopband, so it can weaken the influence of instantaneous random fluctuations on signal trend, reflect local variation characteristics, and highlight the overall change rule.

Based on the weighted moving statistics method, a method for evaluating the nonstationary degree of signals is proposed in this study. According to the different precision requirements of signal analysis, the method appropriately selects and adjusts the weight value and weight range, and the parameters such as mean value, variance and standard deviation, coefficient of variation, crossing-zero rate, and Hurst exponent are chosen as the moving statistics. Through the analysis and comparison of the above statistics, the fluctuation and mutation of signals can be obtained, and the original signals can be characterized clearly and quantitatively. Meanwhile, the occurrence time, sequence, severity, and development process of signal feature changes can be described in details, so as to evaluate the local and overall nonstationary degree of signals. The method is simple and practical and has good applicability, sensitivity, and antinoise.

3.1. Moving Mean Value and Moving Standard Deviation. Weighted moving mean value is the most basic statistic representing the rule of signal moving change. For a certain data point of the signal, the weighted moving average calculation can be carried out for the data itself and the adjacent data points, and the corresponding weighted moving mean value can be obtained as follows:

$$
m_{i}=\frac{1}{1+2 \sum_{k=1}^{m}(1-\alpha)^{k}} \sum_{j=i-m}^{i+m}(1-\alpha)^{|i-j|} x_{j} \text {, }
$$

where $x_{i}$ is the value at the middle point of signal points which needs to be considered for weighting, $m$ is the number of the points participating in the weighting calculation on both sides of the time axis, $x_{j}$ is the value of signal points that need to be considered, $1-\alpha$ is the first-order weight, $m_{i}$ is the moving average at point $i$, and $k$ is the order of weight at $k$ point apart from $x_{i}$.

The moving standard deviation is the standard deviation of the signal processed by weighted moving method, which reflects the degree of data dispersion considering the influence of adjacent signals in the specified interval. The corresponding formula is as follows:

$$
\sigma_{i}=\sqrt{\frac{1}{2 m+1} \sum_{j=i-m}^{i+m}\left(x_{j}-m_{i}\right)^{2}},
$$

where $\sigma_{i}$ is the moving standard deviation at time point $i$, and other parameters have the same meaning as in (11).

The signal itself is influenced by complex factors and internal correlation. The idea of exponential weighting is to give larger weight to the nearer data and smaller weight to the farther data. It maintains the authenticity of the original signal and can effectively reveal the overall change characteristics and local mutation size of the signal.

3.2. Moving Coefficient of Variation. Coefficient of variation refers to the ratio of standard deviation to its average value, which is equal to standardization according to its average value. It can indicate the degree of signal dispersion just like standard deviation. When the units of measurement or the amplitude of multiple signals are different, it is not comprehensive to use the moving standard deviation to represent the dispersion degree or nonstationary degree of different signals, while the moving coefficient of variation can be used for more particular and objective comparison. Based on moving mean value and moving standard deviation, the concept of moving coefficient of variation is 
proposed. It is used to characterize the change of local discrete characteristics and local stationary degree of signals and to analyze the change trend of signals and determine the nonstationary degree of signals. In addition, two kinds of improved moving variation coefficients are proposed according to different engineering requirements in this study.

Moving variation coefficient $\mu$ is the ratio of moving standard deviation to moving mean value:

$$
\mu_{i}=\frac{\sigma_{i}}{m_{i}}
$$

where $m_{i}$ is the moving mean value at point $i$ and $\sigma_{i}$ is the moving standard deviation at point $i$.

In the actual calculation process, since sometimes the moving average appears to be zero or a very small value, it is easy to cause the calculation results of the moving coefficient of variation to not converge, so it cannot be directly used to judge the change law of the random process. Therefore, the formula of moving coefficient of variation is adjusted appropriately, and two improved moving coefficients of variation $\mu_{1}$ and $\mu_{2}$ are proposed, which express the fluctuation and mutation of a stochastic process. The formulas are as follows:

$$
\begin{aligned}
& \mu_{1 i}=\frac{\sigma_{i}}{\lg \left(10^{a m_{i}}+1\right) / a}, \\
& \mu_{2 i}=\frac{\sigma_{i}}{b \cdot \max \left(\left|m_{i}\right|\right)+\left|m_{i}\right|},
\end{aligned}
$$

where $a$ and $b$ can be determined according to the specific characteristics of the signal, and $a$ can be set to a larger value. The value of $b$ is set to make the denominator deviate from zero, so that the coefficient of variation converges; thus, smaller value should be selected.

3.3. Moving Hurst Exponent. The moving Hurst exponent is obtained by setting moving dimension $m$ and defining the effective moving interval of any point $i$ as $[i-m, i+m]$ and calculating the Hurst index in the interval on the basis of traditional Hurst index calculation method. Finally, the exponential curve of the whole duration can be obtained. According to (7), the moving average Hurst exponent is calculated as follows:

$$
H_{i}=\frac{\log \left((R / S)_{l}\right)-\log (K)}{\log (l)} \quad i=m+1, \ldots, n-m .
$$

The moving Hurst exponent can indicate the change of a second-order statistic in the time-varying process. It has the ability to express the similarity of the previous part of signal and the latter part of signal and can intuitively describe the nonstationary degree change characteristics in the process of signal change.

3.4. Moving Crossing-Zero Rate. The effective moving interval $[i-m, i+m]$ is selected and the moving crossing-zero rate $P_{i}$ at point $i$ is obtained by calculating the crossing-zero rate of the interval. The crossing-zero rate of different moving intervals can be obtained by continuously moving the position of moving intervals. The calculation method is as follows:

$$
P_{i}=\frac{N_{i}}{2 m+1} \quad i=m+1, \ldots, n-m,
$$

where $i$ is the position of the center of the rectangular window, $P_{i}$ is the crossing-zero rate at point $i$, and $N_{i}$ is the total number of zero-crossing in the moving interval.

With the change of the moving interval, the moving crossing-zero rate reveals the change rule of frequency characteristics at different time to a certain extent. By extracting the time-varying characteristics of random signals, the change of nonstationary degree of signals can be judged.

\section{Comparison of Signal Nonstationary Degree Evaluation Methods}

In order to compare the ability of traditional methods and moving statistical calculation method to evaluate the signal nonstationary degree, mechanical fault signals, heart disease signals, and several groups of sinusoidal signals mixed with white noise are selected, and the above methods are applied respectively to determine the characteristics and applicability of different nonstationary degree evaluation methods. Among the three signals, sinusoidal signal is the simplest one. Any complex signal can be regarded as the superposition of many sinusoidal signals with different frequencies and amplitudes. The other two signals are the common complex signals in the mechanical field and the biological field. By taking these three signals as examples for verification analysis, the applicability of the proposed methods for simple signals and complex signals and general applicability in different fields can be tested to some extent.

In addition, for the signal whose mean value is not zero and the nonstationarity is not strong, the nonstationary degree analysis can be carried out after subtracting the mean value. This method has no great influence on the accuracy of the calculation results. For the signal whose overall mean value is not zero and the nonstationarity is very strong, the signal itself has a very obvious trend term, so the difficulty of nonstationary degree analysis is lower than that of the signal whose mean value is zero, so this paper does not select the signal whose nonstationary evaluation is less difficult to analyze.

It is worth noting that in this study, $\alpha$ is taken as 0.1 , and the number $m$ of points on the two sides of the data point participating in the weighting calculation is equal to 5 . For other different signals, the values of $\alpha$ and $m$ need to be determined tentatively according to professional needs and signal sampling rate. In general, the value range of $\alpha$ should be between 0 and 0.2 , and the value range of $m$ should be between $1 / 100$ and $1 / 10$ of the signal sampling rate.

4.1. Analysis of Sinusoidal Superposition White Noise Signal. Firstly, nonstationary analysis of sinusoidal signals mixed with white noise is carried out to test the antinoise ability of 
various evaluation methods and the ability to characterize the periodic change of signals. The signal-to-noise ratio of the selected sinusoidal superimposed white noise signal is 30.24 . The unit root value of the signal is -1.11 , and the results show that the test statistic value is larger than the corresponding threshold value of Durbin-Watson (DW) Statistic, so it accepts $H_{0}$, indicating that the signal has unit root, which is a nonstationary stochastic process. The nonstationary degree of sinusoidal superimposed white noise signal is analyzed, and the results are shown in Figures 2 and 3.

In Figure 2, the value attenuation of the autocorrelation function fluctuates slowly and regularly, indicating that the signal is stable and periodic. From the moving crossing-zero rate, it can be seen that the signal has periodicity, but the characteristics of high-frequency noise change are not expressed. The stationary changes of moving Hurst exponent and HHT stationarity indicate that the signal is stationary. In Figure 3, the moving mean reveals the periodic fluctuation of the signal. Because the variation of moving standard deviation is small, it can be judged that the signal is stable. Whenever the original signal reaches the troughs, the moving coefficient of variation $\mu_{1}$ fluctuates greatly, so it can be seen from the moving coefficient of variation $\mu_{1}$ that the signal has periodicity. The position when the original sinusoidal signal fluctuates at the maximum speed is the same as the position when the moving coefficient of variation $\mu_{2}$ is abrupt. However, due to the randomness of white noise, the magnitude of the mutation is not the same, and the sensitivity to noise is highlighted in the moving coefficient of variation $\mu_{2}$. Hence, the moving coefficient of variation has the ability to express periodic changes and mutations accurately and strongly.

4.2. Analysis of Mechanical Fault Signal. The vibration signal of mechanical equipment has rich frequency components, it has typical nonstationarity and nonlinearity, and there is interference, coupling, and transient response among various excitations. Traditional methods usually fail to locate faults correctly. In order to diagnose various abnormal or fault states of mechanical equipment in time and accurately and prevent or eliminate them, it is necessary to analyze the characteristics of vibration signals of equipment. The typical signals from the bearing flaw detection test data published on the website of the bearing data center of Western Reserve University are studied [38]. The vibration signals are composed of three different sets of mechanical fault damage signals, each of which has a length of 1000 data points.

The unit root test of selected mechanical fault signals shows that the unit root values of the three fault signals and their combinations are $-7.48,-7.64,-11.7$, and -14.1 , respectively. All of them reject the original hypothesis at a significant level of $1 \%$. That is, there is no unit root in the four groups of signals, which are a stationary random process. But these signals have obvious nonstationarity on the surface. It can be seen that the unit root test method is prone to miscarriages in the analysis of local stationary degree of signals, which need to be combined with other methods.
Other typical nonstationary degree algorithms are used to analyze the signal nonstationary degree. The analysis results are shown in Figures 4 and 5. The types of mechanical faults can be distinguished by several typical algorithms. The stationarity by HHT can clearly distinguish different types of signal characteristics, and the moving Hurst exponent also has a good effect. Similarly, the results of moving average and moving standard deviation also highlight the nonstationary variation characteristics of the original signal, and the moving variation coefficient $\mu_{2}$ is more powerful in characterizing the local nonstationary degree of the signal.

4.3. ECG Signal Analysis. Heart disease is one of the most serious diseases that threaten human health. Its diagnostic analysis is mainly based on the electrocardiogram (ECG) recording signals. Heart rate variability refers to the time difference between primary and secondary heartbeats, which can be used to predict sudden cardiac death, to evaluate the balance of the cardiac autonomic nervous system and the related pathological state. In this study, three ECG signals from the MIT-BIH arrhythmia database [39] for patients with different heart diseases are chosen to form a set of signals, each of which has 2048 data points in length. As can be seen from the graph, there is no obvious visual difference in the signal of three different lesions in the combined signal, and it is difficult to visualize the heart rate variability and disease results through the naked eye. The ADF test of the selected ECG signals shows that the root values of the three fault signals and their combinations are $-13.46,-10.57$, -6.96 , and -16.0 , respectively. The original hypothesis is rejected at a significant level of $1 \%$. It is considered that the four groups of signals do not have unit roots and are stationary random processes, and misjudgment still occurs.

In order to accurately diagnose the time of cardiac variation and distinguish different symptoms in the actual analysis process, the nonstationary degree of ECG combined signals was analyzed by using various signal nonstationary degree evaluation methods. The results are shown in Figures 6 and 7.

From the analysis results, it can be seen that different evaluation methods have significant differences in distinguishing ability. Compared with the distinguishing ability of mechanical fault signals, the stationary degree based on HHT and the moving Hurst exponent make it not easy to distinguish different groups of signals, but moving crossingzero rate has better distinguishing ability, which can judge the time of variation of cardiac signals. The moving mean value and the moving standard deviation make the change characteristics of the original signal prominent. The performance of moving coefficient of variation is the most outstanding. Moving coefficient of variation $\mu_{1}$ can distinguish different variation states in order to get the time of change. Moving coefficient of variation $\mu_{2}$ can get the heart rate variability and the time point and size of heart mutation, which is helpful for professionals to diagnose arrhythmia, myocarditis, and other symptoms.

In order to analyze the influence of window length on the calculation results, taking the sinusoidal signal, mechanical 


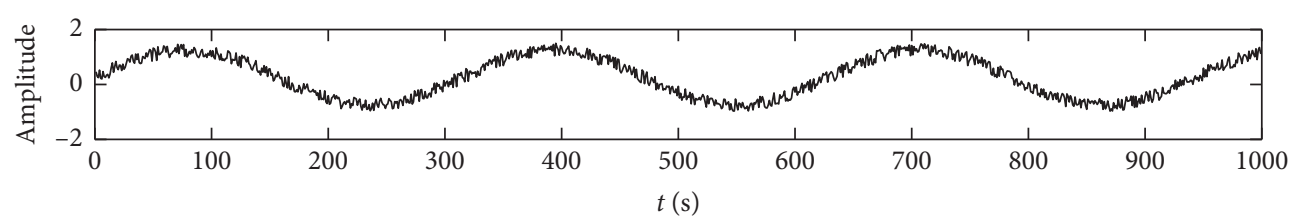

- Original wave

(a)

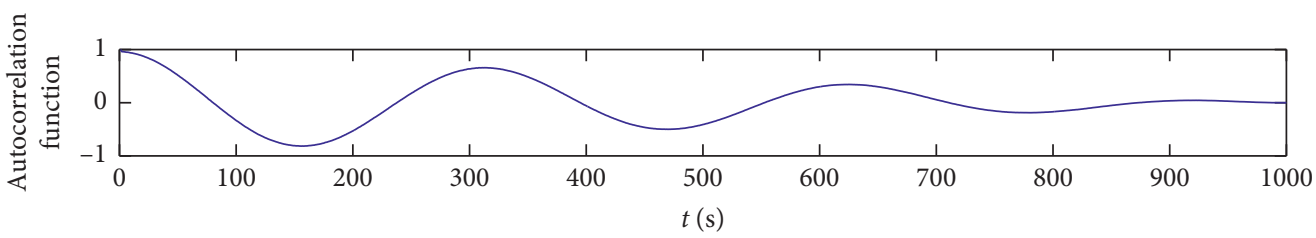

— Autocorrelation function

(b)

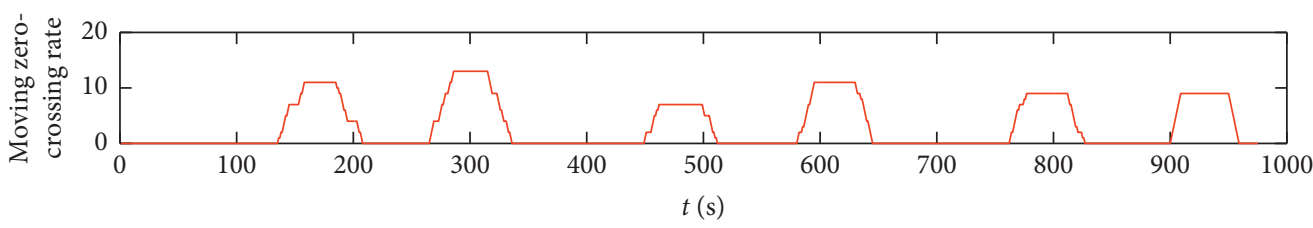

__ Moving zero-crossing rate

(c)

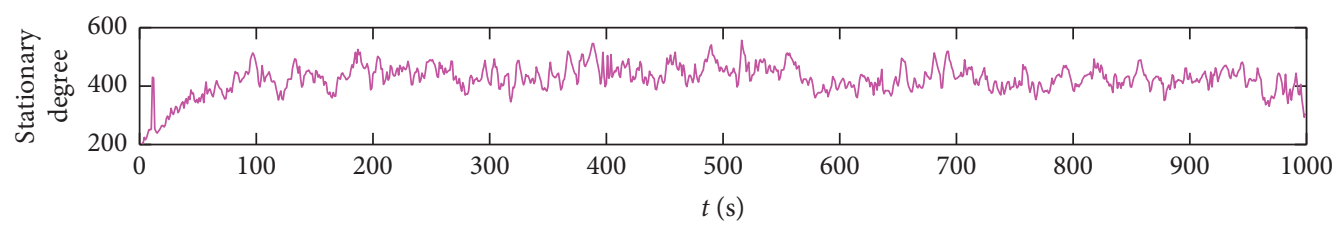

— Stationary degree

(d)

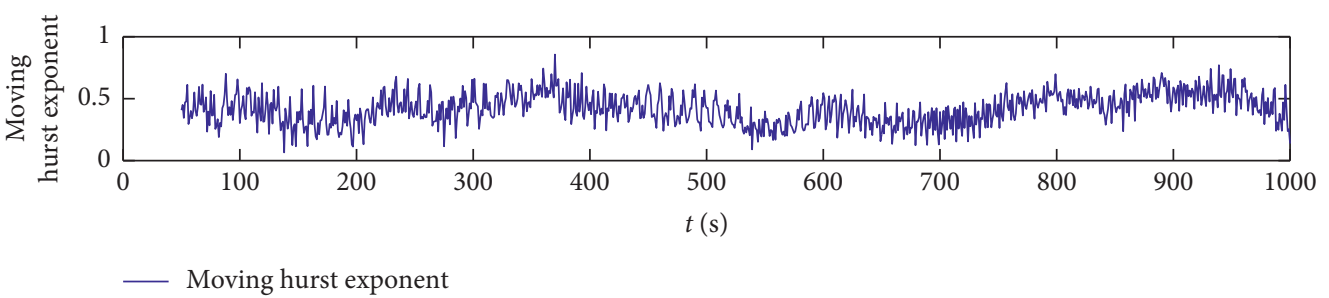

(e)

FIGURE 2: Analysis of sinusoidal superimposed white noise signal based on typical algorithms.

fault signal, and ECG signal as examples, the nonstationary degree of the signal under different window lengths $(m=1$, $5,9,13)$ is analyzed by the moving coefficient of variation method. The results obtained are shown in Figures 8 to 10. As can be seen from the figures, the selection of window length has a very important influence on the calculation results. When the value of the window length is within a reasonable range, the calculation results are not much different; when the value of the window length is large, there will be a certain error in the calculation results, and the accuracy will be reduced. In addition, when the value of the window length is large, the calculation result will be excessively smooth, and some time-varying details of signal changes will be lost. Therefore, the value range of the window length needs to be reasonably limited according to the actual situation and calculation accuracy.

4.4. Comparison of Signal Recognition Ability Based on Different Nonstationary Degree. The above example analysis mainly focuses and compares the identification ability of different nonstationary degree analysis methods. In practical application, it is often necessary to realize the automation, real-time, and intelligence of signal classification or fault diagnosis according to engineering requirements. Therefore, it is usually necessary to use the pattern recognition method to classify and recognize signals quickly and accurately 


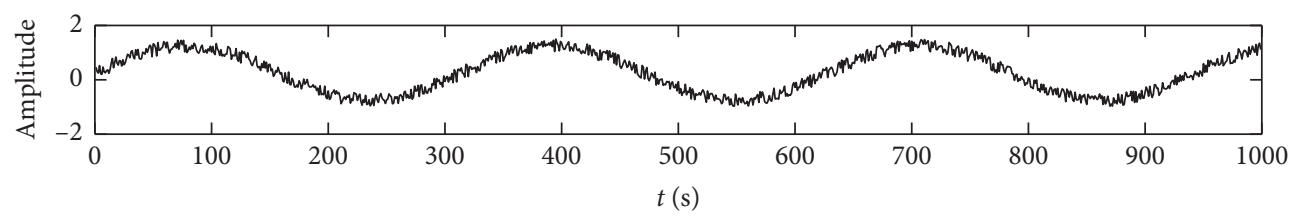

— Original wave

(a)

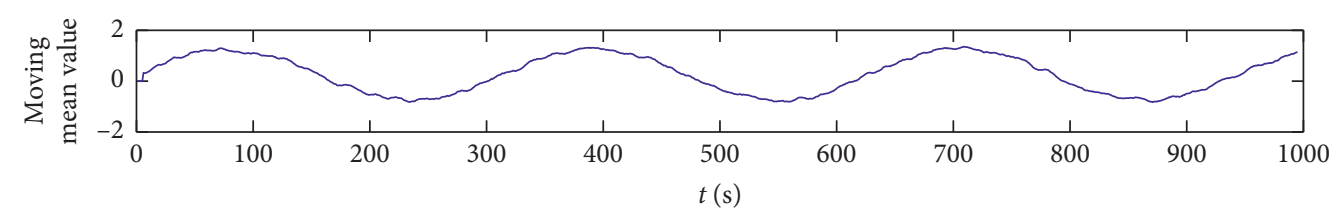

— Moving mean value

(b)

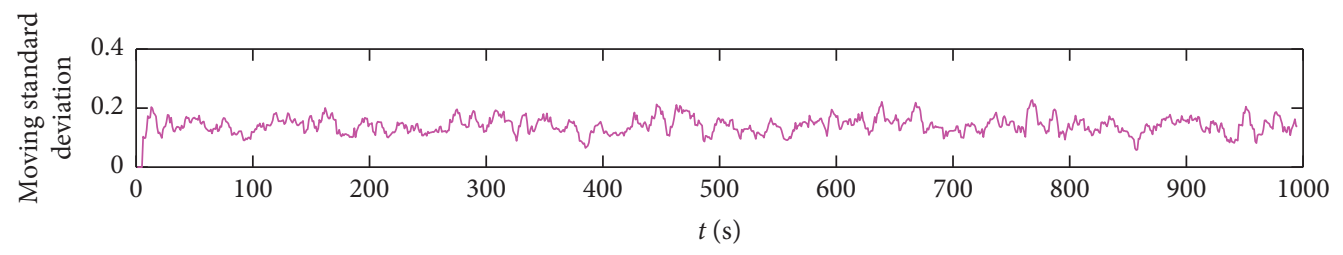

— Moving standard deviation

(c)

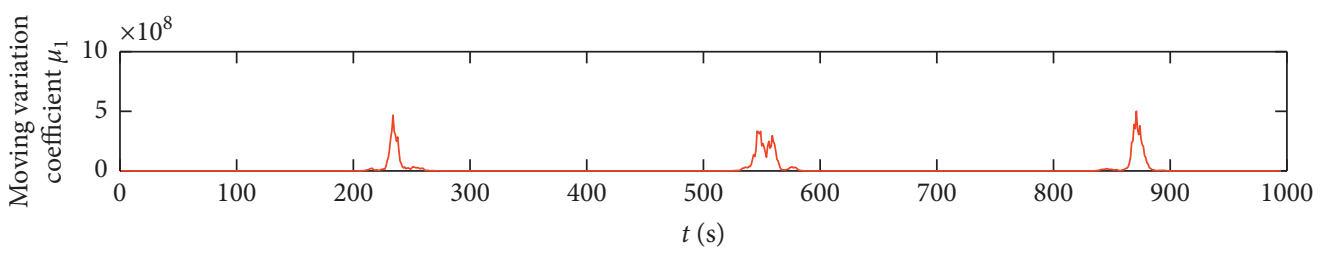

(d)

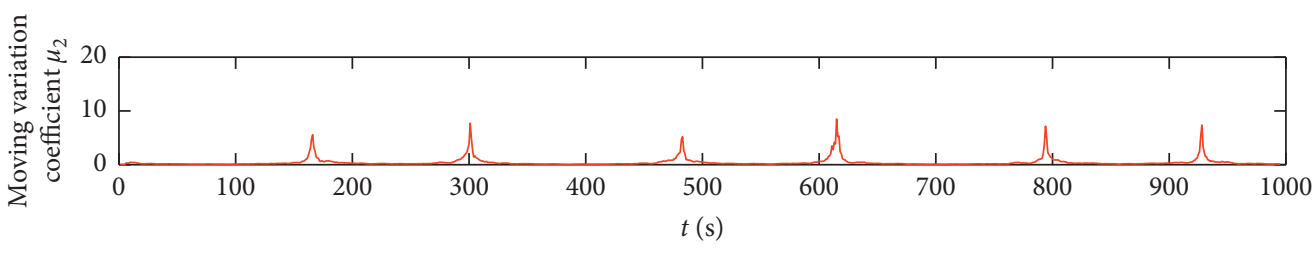

— Moving variation coefficient $\mu_{2}$

(e)

FIgURE 3: Analysis of sinusoidal superimposed white noise signal based on moving algorithms.

according to their characteristics. If nonstationary degree evaluation method is used to extract signal features and recognize patterns, it is necessary to select excellent pattern parameters and establish effective feature vectors.

On the basis of the above research, the moving crossing-zero rate, HHT time-domain stationary degree, the moving Hurst exponent, and the moving coefficient of variation $\mu_{1}$ and $\mu_{2}$ with strong discriminating ability are selected as the model parameters. Due to the signal energy, the sum of square of signal amplitude is the basic characteristic of the signal and its physical meaning is clear; the mode parameters of different parts of the mechanical fault signal and ECG signal are obtained and corresponding total signal energy is calculated. In order to eliminate the dimension effect of different parameters, the total energy of each parameter is normalized. The results are shown in Figures 11 and 12, and the discrimination ability is compared. 


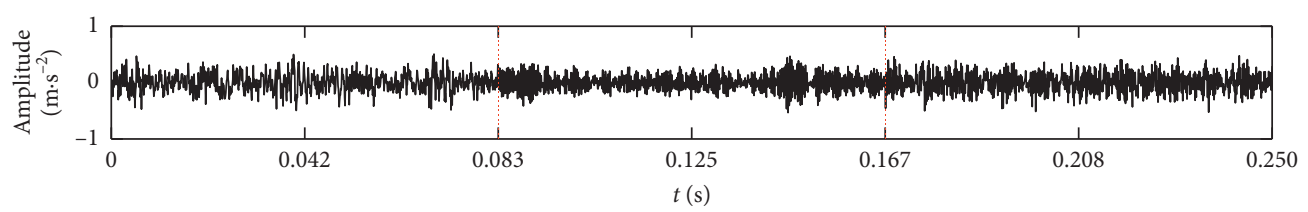

(a)

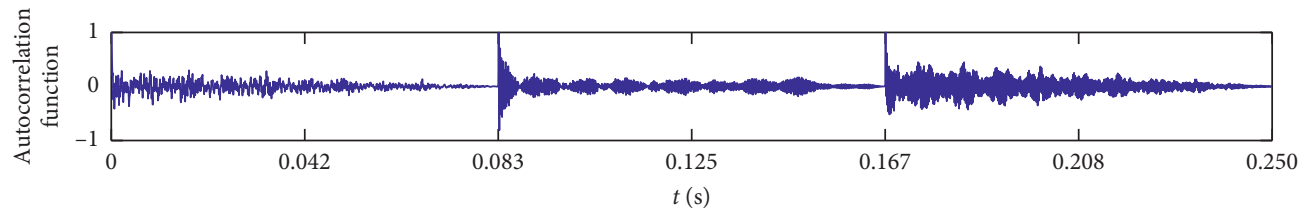

— Autocorrelation function

(b)

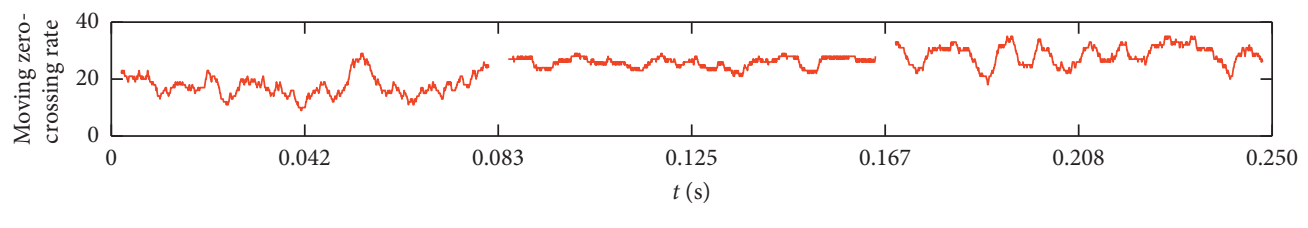

__ Moving zero-crossing rate

(c)

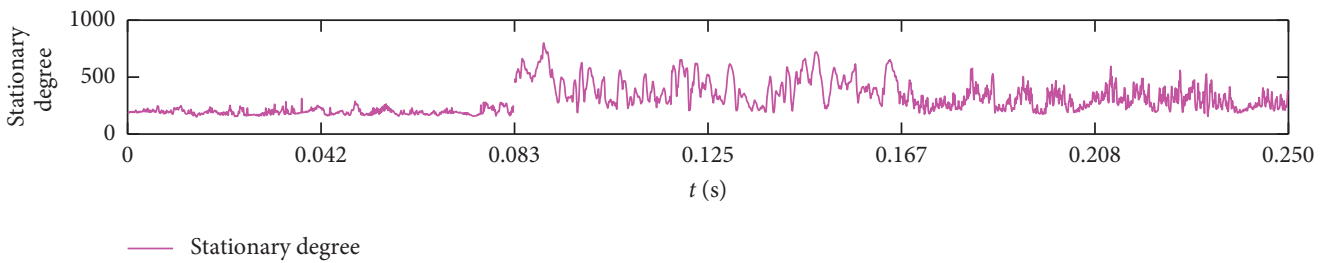

(d)

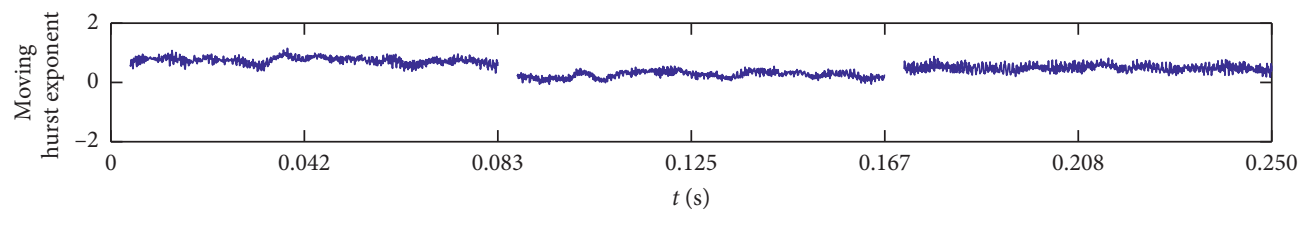

_- Moving hurst exponent

(e)

Figure 4: Analysis of mechanical fault signals based on typical algorithms.

The results show that in the analysis of mechanical fault signal and ECG signal, the signal energy of HHT stationary degree, moving mean value, and moving coefficient of variation $\mu_{2}$ all show good discrimination. Meanwhile, the moving crossing-zero rate, moving Hurst exponent, moving standard deviation, and moving coefficient of variation also show good discriminant ability in the analysis of ECG signal.

Based on the above analysis, it can be concluded that HHT stationary degree, moving mean value, and moving coefficient of variation $\mu_{2}$ can be widely used in different types of signal analysis. In addition, the proposed moving crossing-zero rate, moving Hurst exponent, moving standard deviation, and moving variation coefficient $\mu_{1}$ also show fine discriminant ability in some signal analyses, so the signal analysis method can be reasonably selected according to the type and characteristics of the signal. On the whole, the signal analysis methods based on the moving statistical idea, such as moving coefficient of variation, can reveal the relationship and change between adjacent signals, adequately display the time-varying characteristics of signals, and have unique advantages in signal analysis and processing. 


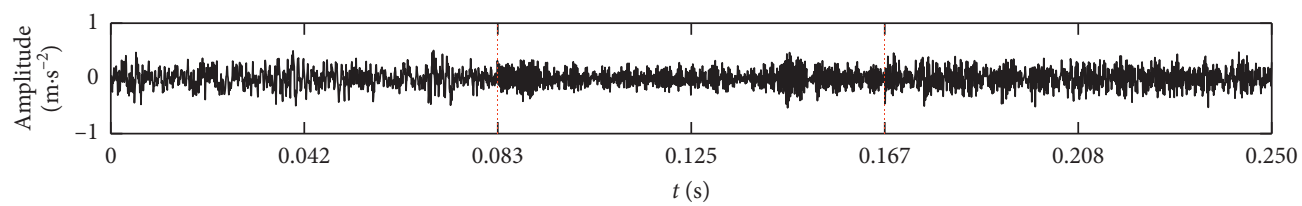

_ Original wave

(a)

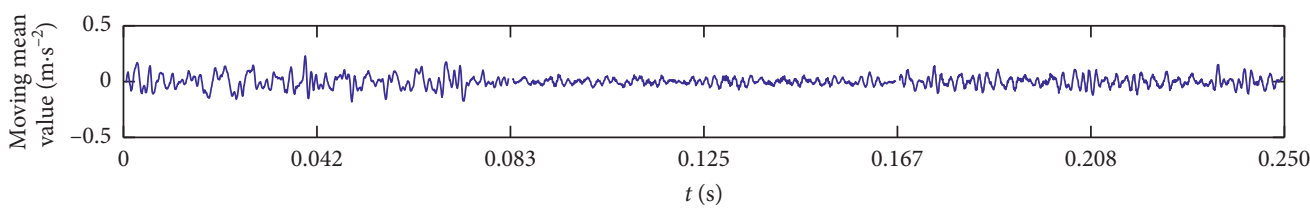

_ Moving mean value

(b)

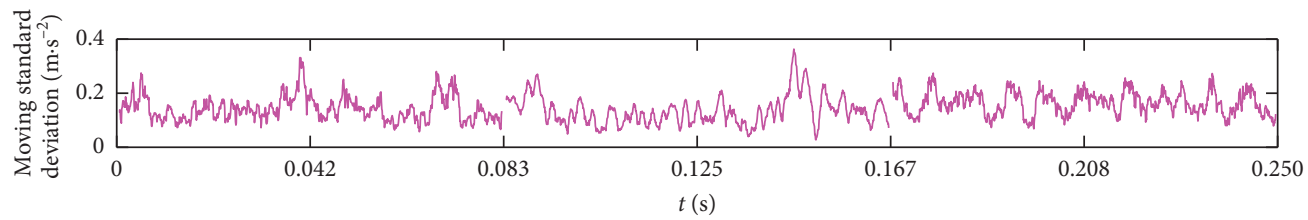

(c)

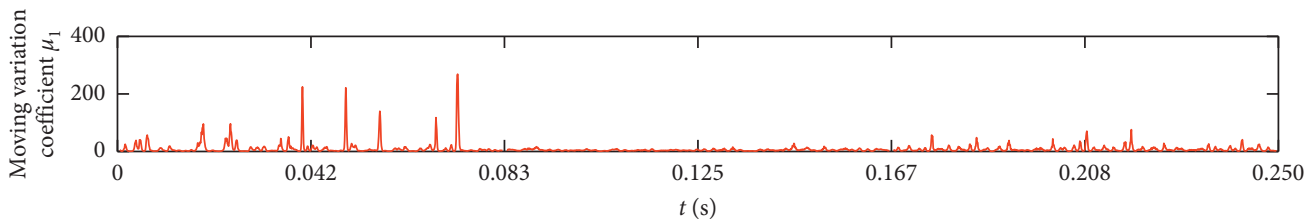

_ Moving variation coefficient $\mu_{1}$

(d)

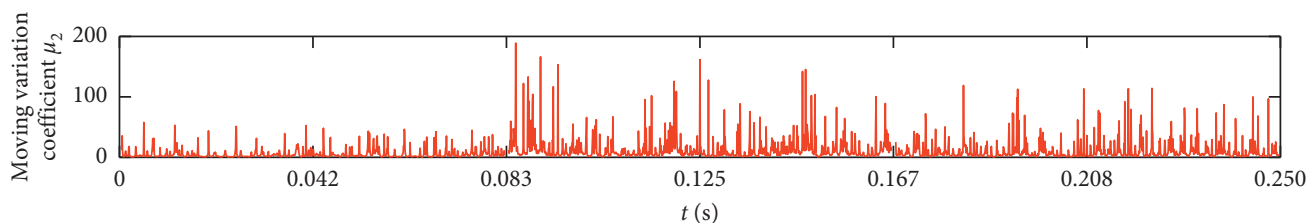

_ Moving variation coefficient $\mu_{2}$

(e)

FIgURE 5: Analysis of mechanical fault signals based on moving algorithms.

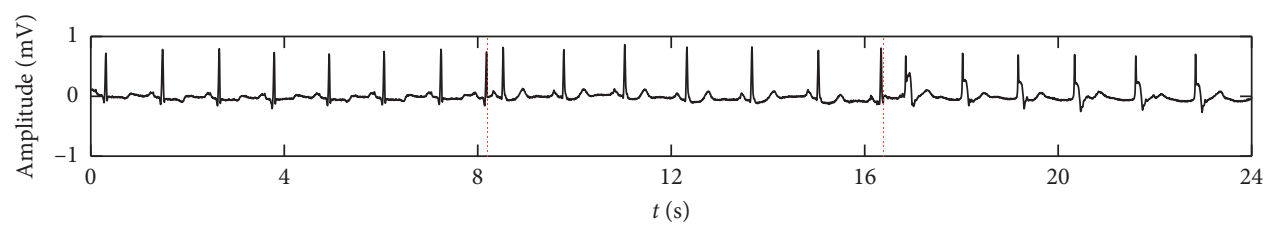

— Original wave

(a)

FIgURE 6: Continued. 


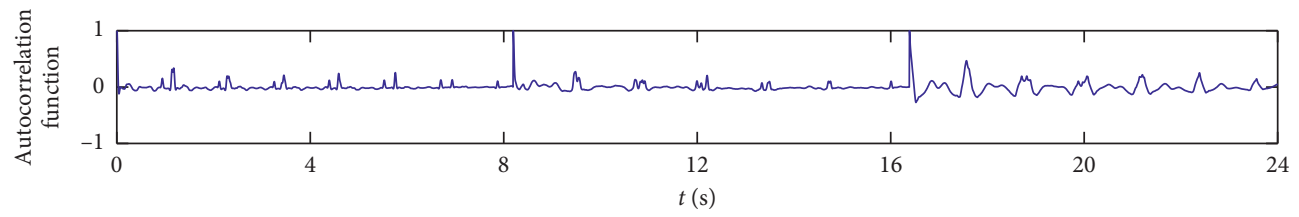

- Autocorrelation function

(b)

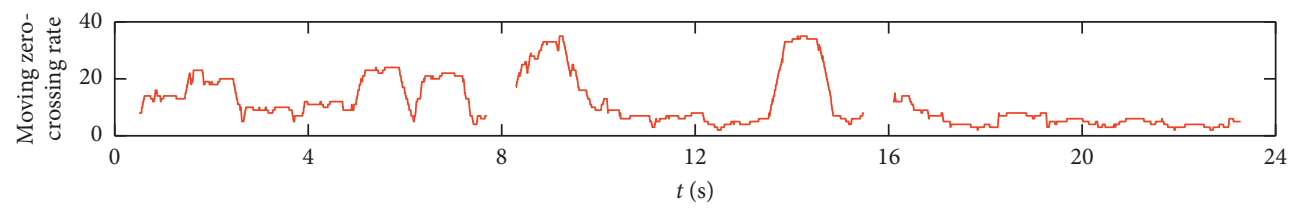

_ Moving zero-crossing rate

(c)

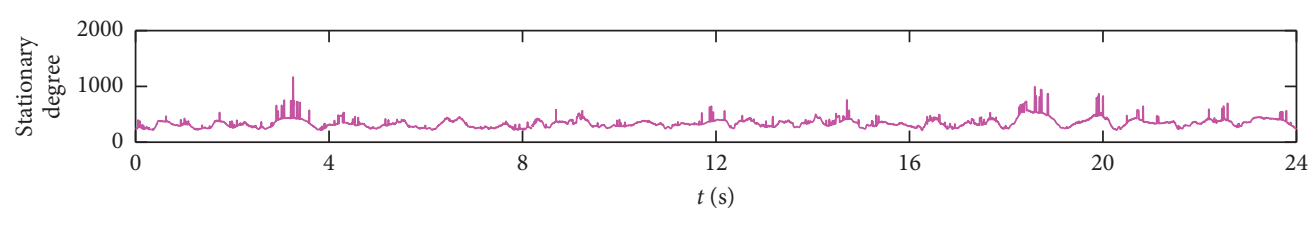

_ Stationary degree

(d)

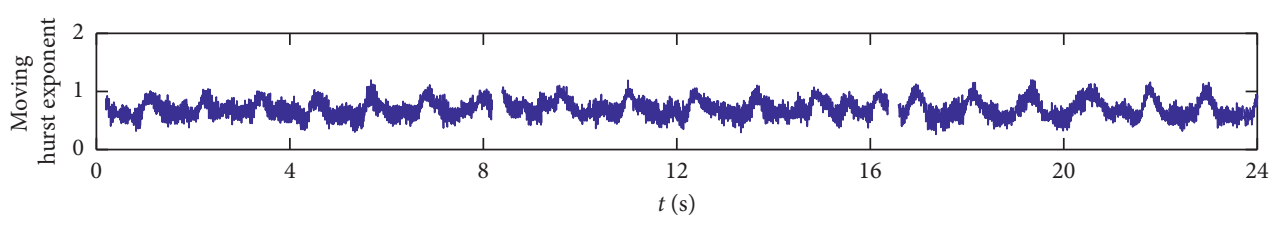

__ Moving hurst exponent

(e)

FIGURE 6: Analysis of ECG signal based on typical algorithms.

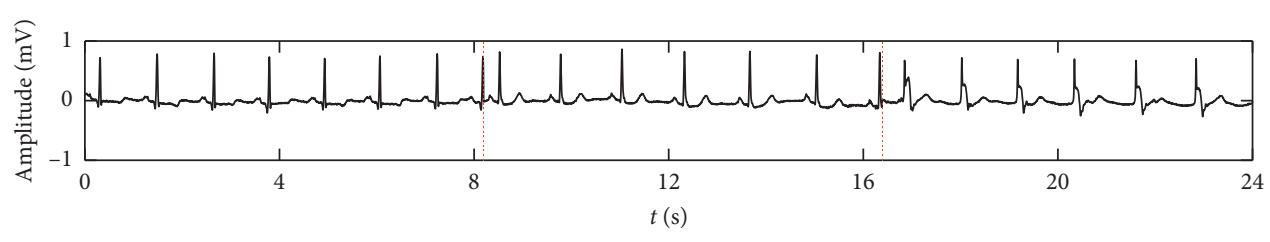

— Original wave

(a)

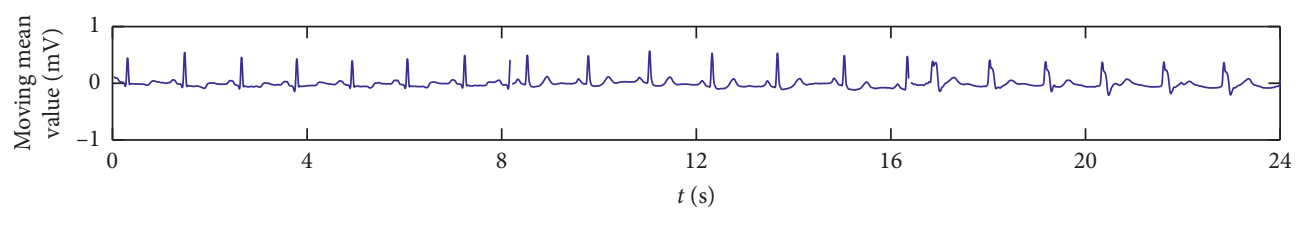

(b)

Figure 7: Continued. 


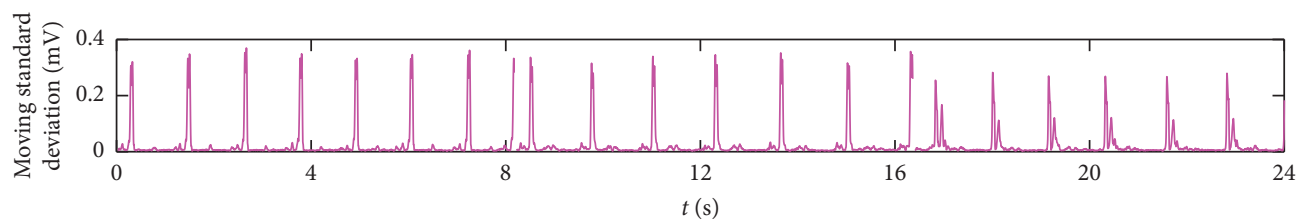

_ Moving standard deviation

(c)

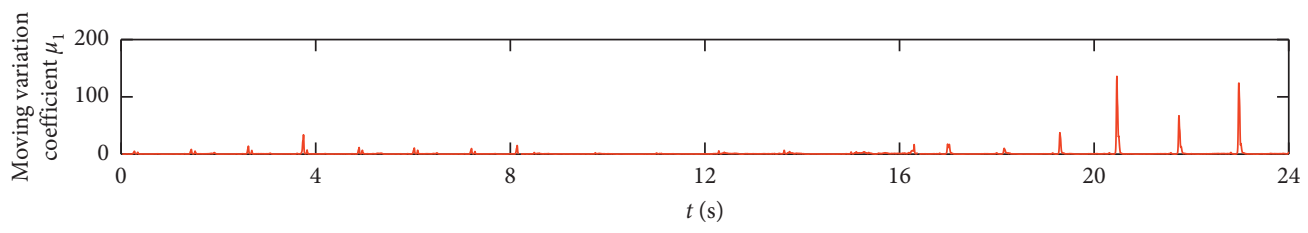

Moving variation coefficient $\mu_{1}$

(d)

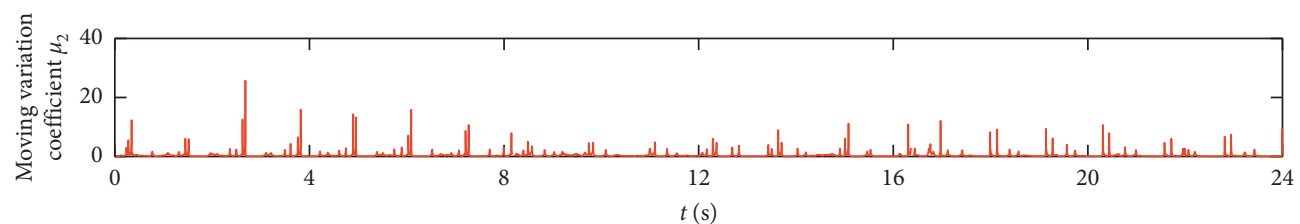

- Moving variation coefficient $\mu_{2}$

(e)

FIGURE 7: Analysis of ECG signal based on moving statistic algorithm.

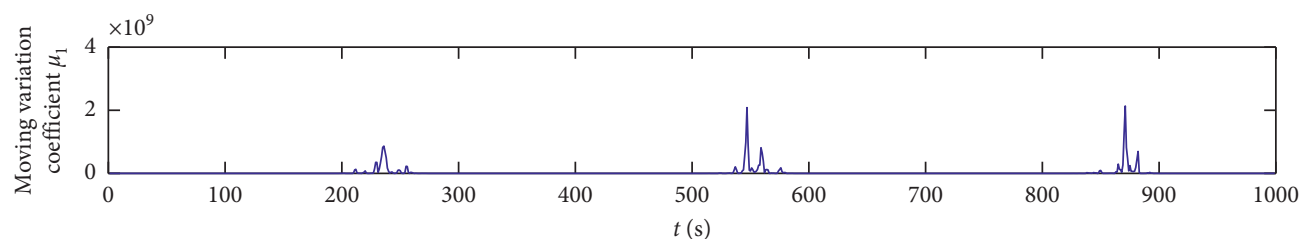

$m=1$

(a)

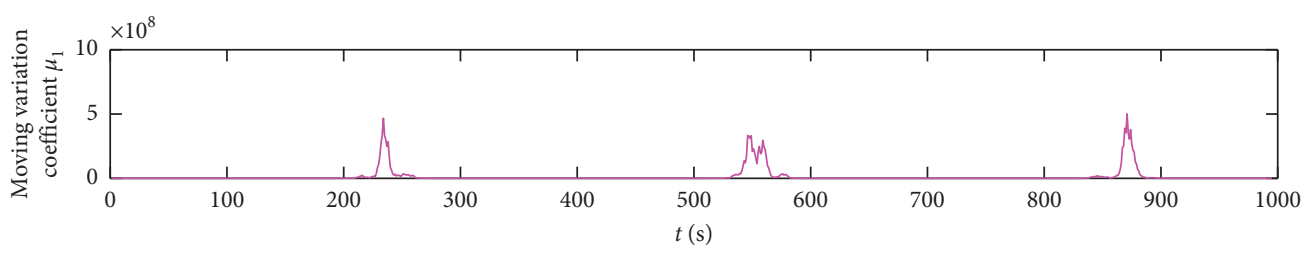

$m=5$

(b)

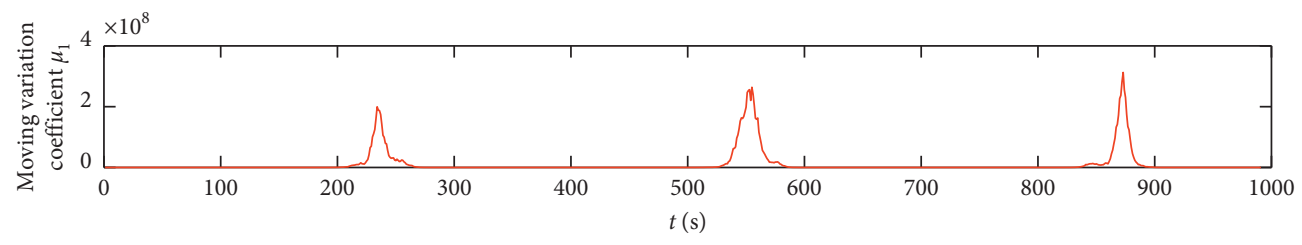

$m=9$

(c)

Figure 8: Continued. 


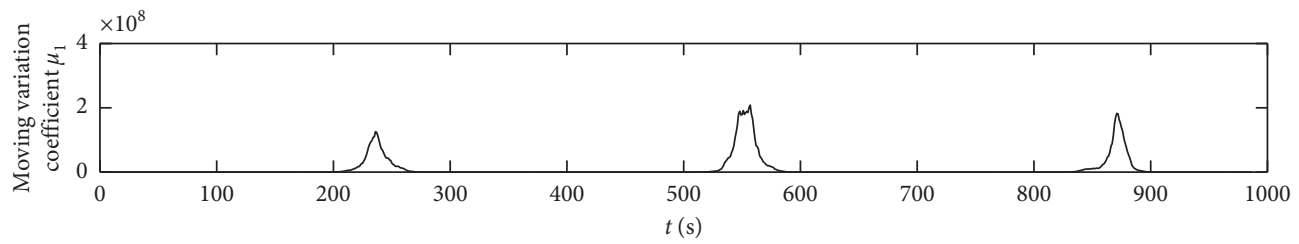

$m=13$

(d)

Figure 8: Calculation results of sinusoidal signal under different window lengths.

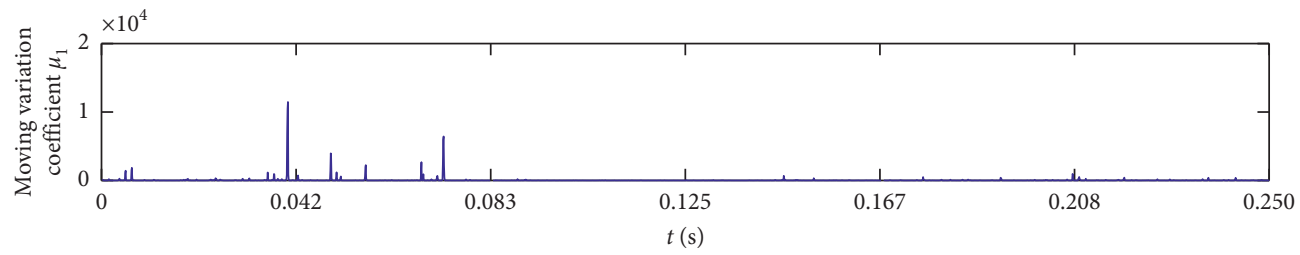

$-m=1$

(a)

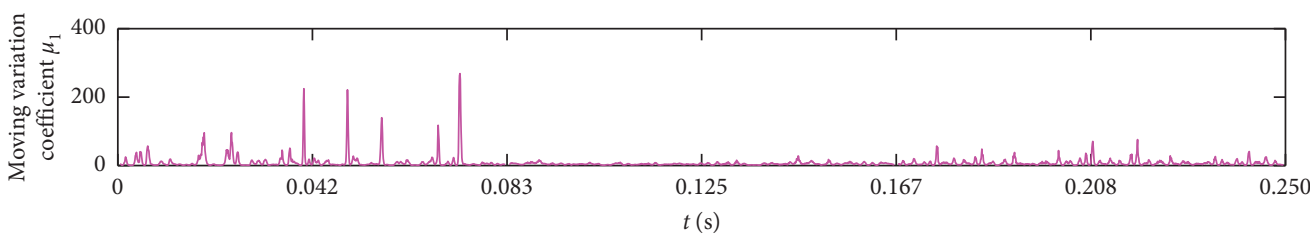

$m=5$

(b)

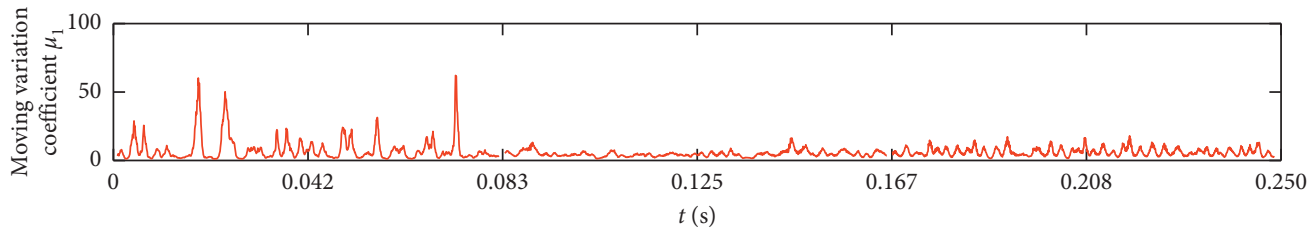

$-m=9$

(c)

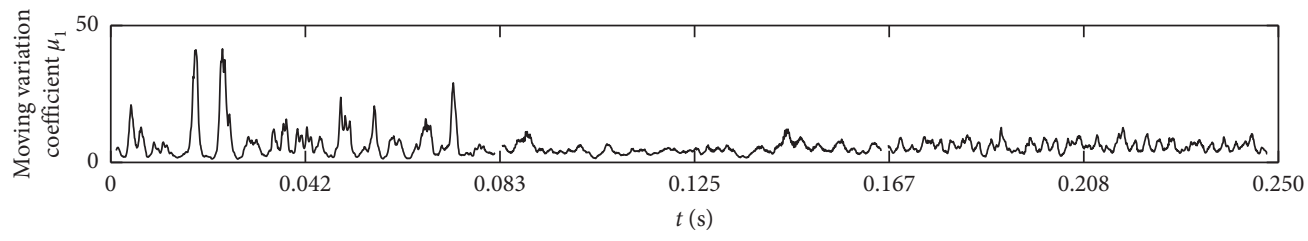

$-m=13$

(d)

FIgURE 9: Calculation results of mechanical fault signals under different window lengths.

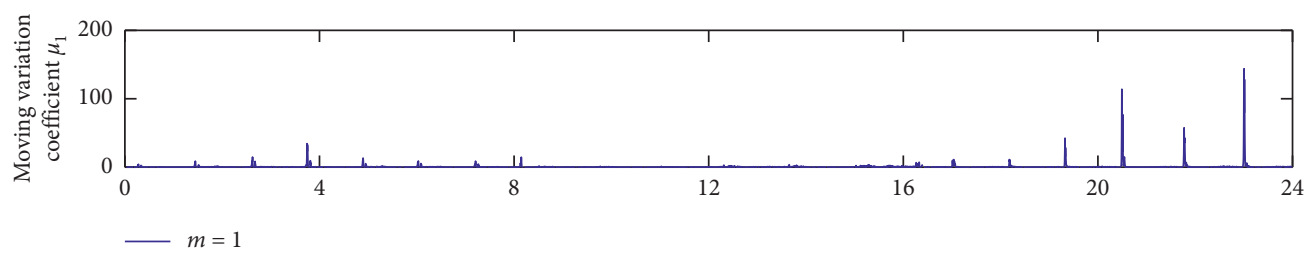

(a)

FIgURE 10: Continued. 


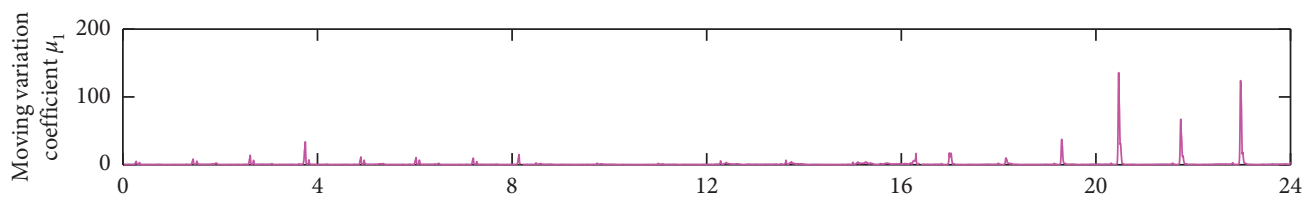

(b)

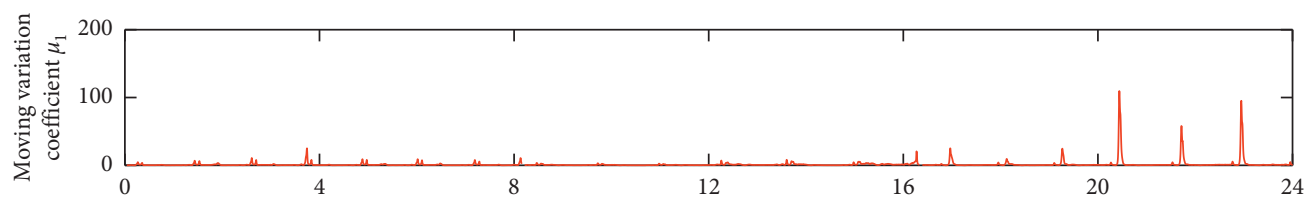

- $m=9$

(c)

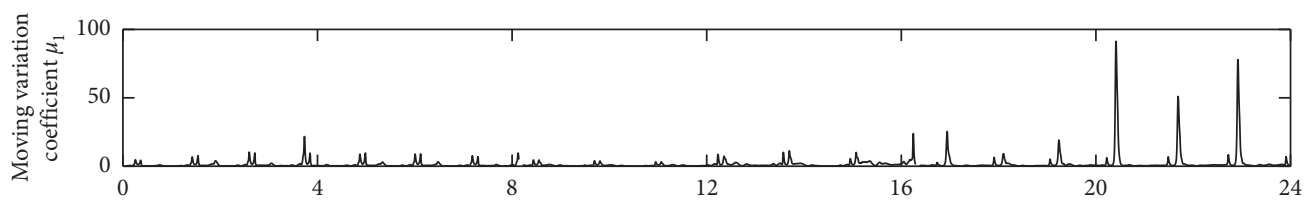

- $m=13$

(d)

Figure 10: Calculation results of ECG signals under different window lengths.

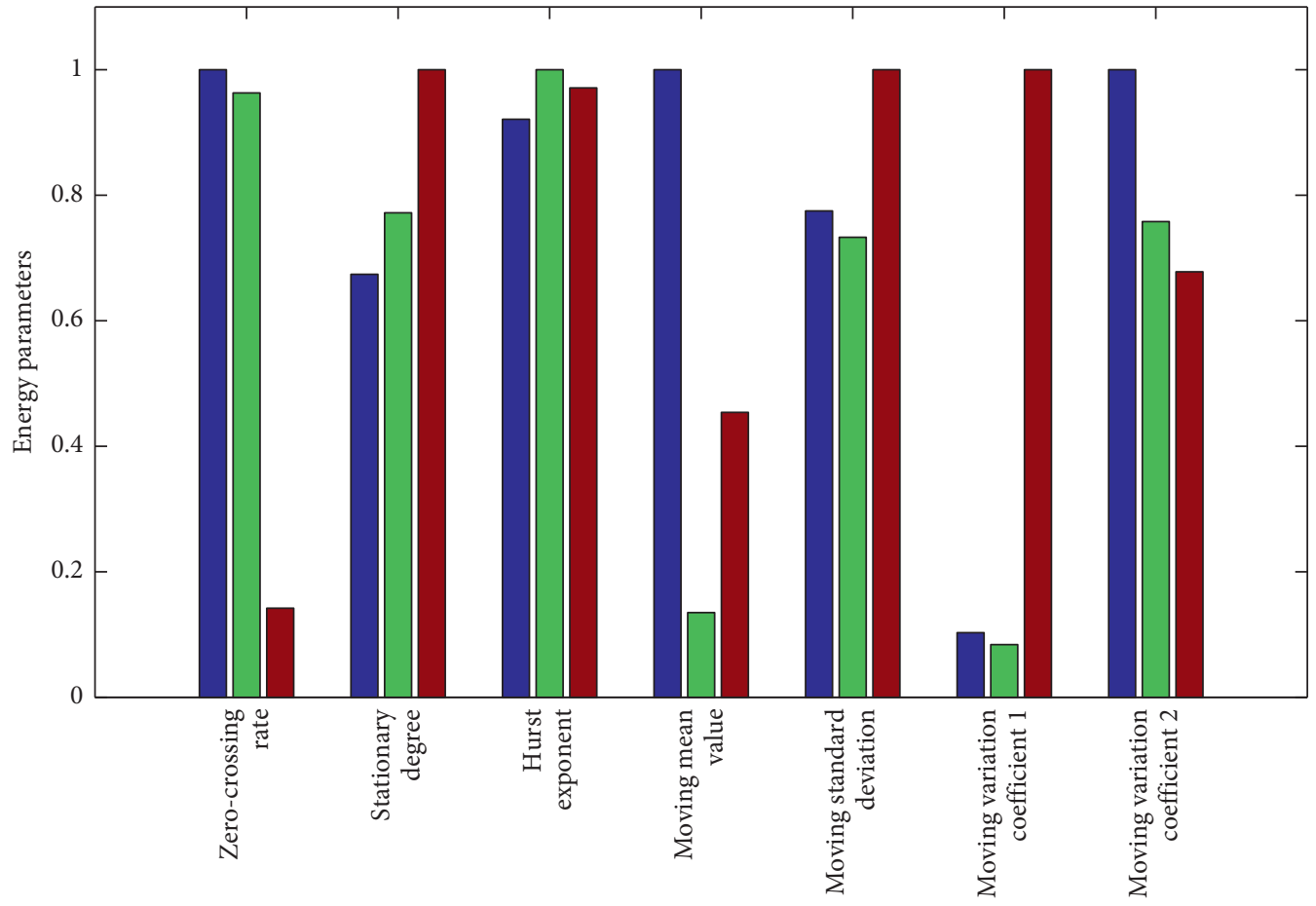

First part

Second part

Third part

Figure 11: Nonstationarity energy index of mechanical fault signal. 


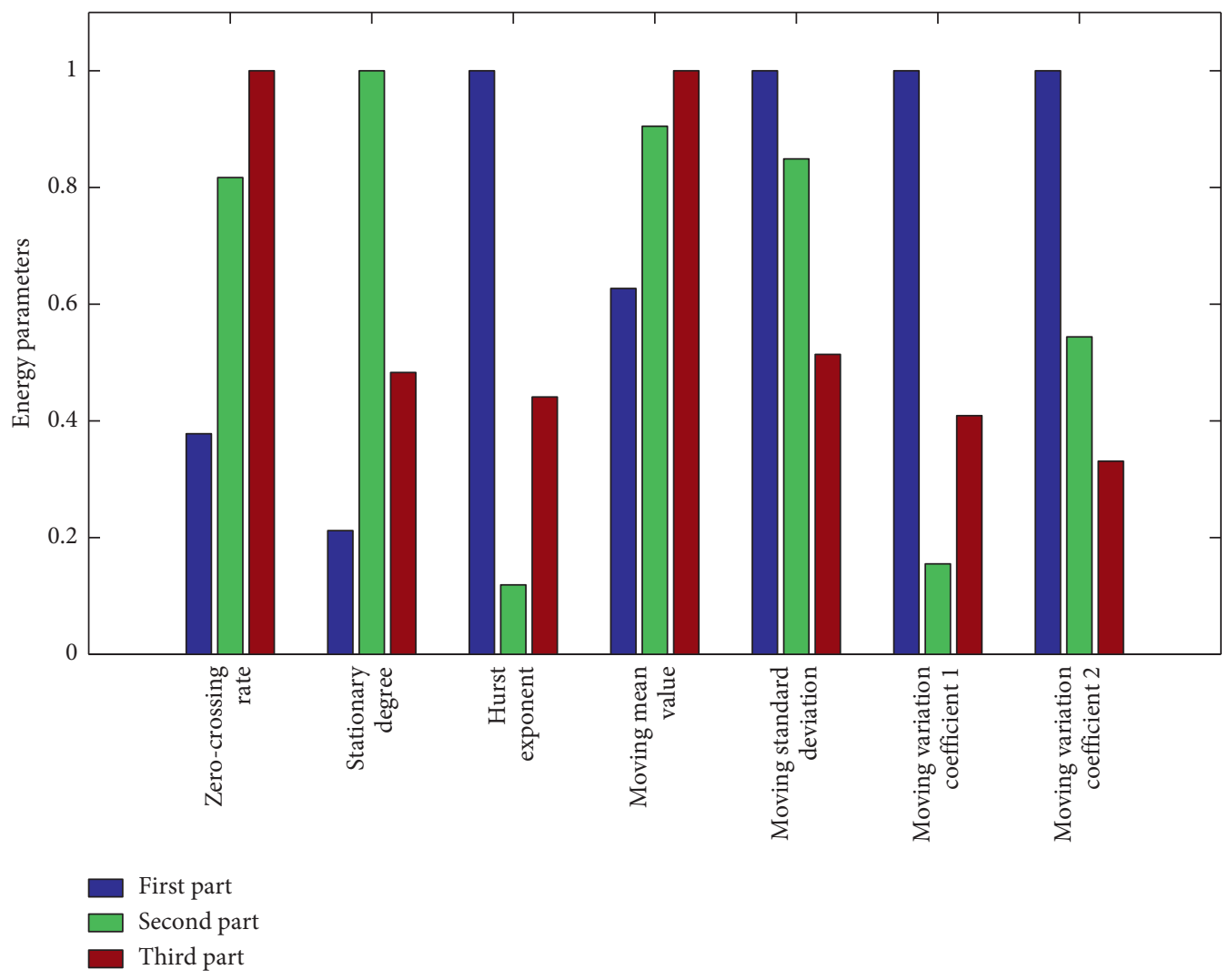

FIgURe 12: Nonstationarity energy index of ECG signal.

\section{Conclusion}

The signal nonstationary degree evaluation is an effective tool for nonstationary signal diagnosis. In view of the limitations of the existing nonstationary evaluation methods in the scope of application, this paper considers the timevarying characteristics of nonstationary signals, and the concepts and calculation methods of moving coefficient of variation and moving Hurst exponent are proposed based on the idea of moving statistical analysis, which can reflect the time-varying details of the signal, so as to provide effective support for the evaluation of signal nonstationary. By analyzing the three different signals of sinusoidal signal, mechanical fault signal, and ECG signal, it can be known that compared with the existing typical nonstationary degree evaluation methods, the signal nonstationary degree evaluation methods such as moving variation coefficient proposed in this paper can be accurate and reliable. It accurately reveals the fluctuation and variation of the signal, can reveal the subtle changes in the local details of the signal, and has a better ability to identify the nonstationary degree of the signal. The evaluation method of signal nonstationary degree based on moving statistical characteristics proposed in this paper can be used in many fields such as fault diagnosis and has important engineering value and significance.

Because some constants in the signal nonstationary degree evaluation method proposed in this paper are empirical constants that need to be determined after preliminary selection based on signal characteristics, in the following research, the authors intend to determine the corresponding constants for different types of signals through a large number of analyses. On this basis, for signals with small differences, the authors will further propose fine characterization parameters or methods that can distinguish the global nonstationary degree.

\section{Data Availability}

The data used to support the findings of this study are available from the corresponding author upon request.

\section{Conflicts of Interest}

The authors declare that they have no known conflicts of interest or personal relationships that could have appeared to influence the work reported in this paper.

\section{Acknowledgments}

This work was partially supported by the National Key R \& D Program of China (Grant nos. 2017YFC1500604 and 2017YFC1500603) and the Natural Science Foundation of China (Grant no. 51878017). 


\section{References}

[1] J. K. Hammond and P. R. White, "The analysis of non-stationary signals using time-frequency methods," Journal of Sound and Vibration, vol. 190, no. 3, pp. 419-447, 1996.

[2] L. Li, H. Cai, H. Han, Q. Jiang, and H. Ji, "Adaptive short-time Fourier transform and synchrosqueezing transform for nonstationary signal separation," Signal Processing, vol. 166, 2020.

[3] F. Adamo, F. Attivissimo, A. Di Nisio, M. Savino, and M. Spadavecchia, "A spectral estimation method for nonstationary signals analysis with application to power systems," Measurement, vol. 73, pp. 247-261, 2015.

[4] H. Zhao, H. Liu, Y. Jin, X. Dang, and W. Deng, "Feature extraction for data-driven remaining useful life prediction of rolling bearings," IEEE Transactions on Instrumentation and Measurement, vol. 70, pp. 1-10, 2021.

[5] A. Glowacz, "Acoustic fault analysis of three commutator motors," Mechanical Systems Signal Processing, vol. 133, 2019.

[6] A. Glowacz, "Recognition of acoustic signals of induction motor using FFT, SMOFS-10 and LSVM," Eksploatacja I Niezawodnosc-Maintenance and Reliability, vol. 17, pp. 569574, 2015.

[7] R. Uma Maheswari and R. Umamaheswari, "Trends in nonstationary signal processing techniques applied to vibration analysis of wind turbine drive train-a contemporary survey," Mechanical Systems and Signal Processing, vol. 85, pp. 296311, 2017.

[8] W. Martin and P. Flandrin, "Wigner-Ville spectral analysis of nonstationary processes," IEEE Transactions on Acoustics, Speech, and Signal Processing, vol. 33, no. 6, pp. 1461-1470, 1985.

[9] Y. F. Tang, F. Lin, and Q. Zou, "Complexity analysis of timefrequency features for vibration signals of rolling bearings based on local frequency," Shock and Vibration, vol. 2019, Article ID 7190568, 2019.

[10] Y. Yang, X. Dong, Z. Peng, W. Zhang, and G. Meng, "Component extraction for non-stationary multi-component signal using parameterized de-chirping and band-pass filter," IEEE Signal Processing Letters, vol. 22, no. 9, pp. 1373-1377, 2015.

[11] Y. Lu and J. Tang, "On time-frequency domain feature extraction of wave signals for structural health monitoring," Measurement, vol. 114, pp. 51-59, 2018.

[12] N. E. Huang, Z. Shen, S. R. Long et al., "The empirical mode decomposition and the Hilbert spectrum for nonlinear and non-stationary time series analysis," Proceedings of the Royal Society A-Mathematical Physical and Engineering Sciences, vol. 454, no. 1971, pp. 903-995, 1998.

[13] S. Mukhopadhyay and P. Sircar, "Parametric modelling of non-stationary signals: a unified approach," Signal Processing, vol. 60, no. 2, pp. 135-152, 1997.

[14] Y. Grenier, "Time-dependent ARMA modeling of nonstationary signals," IEEE Transactions on Acoustics, Speech, and Signal Processing, vol. 31, no. 4, pp. 899-911, 1983.

[15] N. Martin, "An AR spectral analysis of non-stationary signals," Signal Processing, vol. 10, no. 1, pp. 61-74, 1986.

[16] D. A. Dickey and W. A. Fuller, "Distribution of the estimators for autoregressive time series with a unit root," Journal of the American Statistical Association, vol. 74, no. 366a, pp. 427431, 1979.

[17] F. Emirmahmutoglu, T. Omay, S. J. H. Shahzad, and S. M. Nor, "Smooth break detection and de-trending in unit root testing," Mathematics, vol. 9, no. 4, 2021.
[18] C. Hanck and R. Czudaj, "Nonstationary-volatility robust panel unit root tests and the great moderation," AStA Advances in Statistical Analysis, vol. 99, no. 2, pp. 161-187, 2015.

[19] R. Marschinski and H. Kantz, "Analysing the information flow between financial time series," The European Physical Journal B, vol. 30, no. 2, pp. 275-281, 2002.

[20] J. De Jesus Rangel-Magdaleno, H. Peregrina-Barreto, J. M. Ramirez-Cortes, P. Gomez-Gil, and R. Morales-Caporal, "FPGA-based broken bars detection on induction motors under different load using motor current signature analysis and mathematical morphology," IEEE Transactions on Instrumentation and Measurement, vol. 63, no. 5, pp. 10321040, 2014.

[21] N. Marwan, M. Carmenromano, M. Thiel, and J. Kurths, "Recurrence plots for the analysis of complex systems," Physics Reports, vol. 438, no. 5-6, pp. 237-329, 2007.

[22] B. M. Mathunjwa, Y. T. Lin, C. H. Lin, M. F. Abbod, and J. S. Shieh, "ECG arrhythmia classification by using a recurrence plot and convolutional neural network," Biomedical Signal Processing and Control, vol. 64, 2021.

[23] Y. Hsueh, V. R. Ittangihala, W. B. Wu, H. C. Chang, and C. C. Kuo, "Condition monitor system for rotation machine by CNN with recurrence plot," Energies, vol. 12, no. 17, 2019.

[24] H. Yang, S. T. S. Bukkapatnam, and L. G. Barajas, "Local recurrence based performance prediction and prognostics in the nonlinear and nonstationary systems," Pattern Recognition, vol. 44, no. 8, pp. 1834-1840, 2011.

[25] A. Carbone, G. Castelli, and H. E. Stanley, "Time-dependent Hurst exponent in financial time series," Physica A: Statistical Mechanics and its Applications, vol. 344, no. 1-2, pp. 267-271, 2004.

[26] G. R. Saragoni and G. C. Hart, "Simulation of artificial earthquakes," Earthquake Engineering \& Structural Dynamics, vol. 2, no. 3, pp. 249-267, 1974.

[27] T. Schreiber, "Detecting and analysing nonstationarity in a time series with nonlinear cross-predictions," Physical Review Letters, vol. 78, no. 5, pp. 843-846, 1999.

[28] T. Graves, R. B. Gramacy, C. L. E. Franzke, and N. W. Watkins, "Efficient Bayesian inference for natural time series using ARFIMA processes," Nonlinear Processes in Geophysics, vol. 22, no. 6, pp. 679-700, 2015.

[29] H. Cao, D. E. Lake, M. P. Griffin, and J. R. Moorman, "Increased nonstationarity of neonatal heart rate before the clinical diagnosis of sepsis," Annals of Biomedical Engineering, vol. 32, no. 2, pp. 233-244, 2004.

[30] I. G. Pechlivanidis, B. Jackson, H. Mcmillan, and H. V. Gupta, "Robust informational entropy-based descriptors of flow in catchment hydrology," Hydrological Sciences Journal, vol. 61, no. 1, pp. 1-18, 2016.

[31] Z. Xu, H. Wang, H. Wan, and H. Li, "Quantitative assessment of nonstationarity of wind speed signal using recurrence plot," Journal of Aerospace Engineering, vol. 32, no. 6, Article ID 04019094, 2019.

[32] Q. Li and S. Y. Liang, "Degradation trend prognostics for rolling bearing using improved $R / S$ statistic model and fractional Brownian motion approach," IEEE Access, vol. 6, pp. 21103-21114, 2018.

[33] S. Mohanty, K. K. Gupta, and K. S. Raju, "Hurst based vibroacoustic feature extraction of bearing using EMD and VMD," Measurement, vol. 117, pp. 200-220, 2018.

[34] Z. Wu and N. E. Huang, "Ensemble empirical mode decomposition: a noise-assisted data analysis method," Advances in Adaptive Data Analysis, vol. 1, no. 1, pp. 1-41, 2009. 
[35] N. E. Huang, Z. Shen, and S. R. Long, "A new view of nonlinear water waves: the Hilbert spectrum," Annual Review of Fluid Mechanics, vol. 31, no. 1, pp. 417-457, 1999.

[36] K. Kumar, S. Shukla, and S. K. Singh, "A combined approach for weak fault signature extraction of rolling element bearing using Hilbert envelop and zero frequency resonator," Journal of Sound and Vibration, vol. 419, pp. 436-451, 2018.

[37] F. Sabbaghian-Bidgoli and J. Poshtan, "Fault detection of broken rotor bar using an improved form of Hilbert-Huang transform," Fluctuation and Noise Letters, vol. 17, no. 2, 2018.

[38] https://csegroups.case.edu/bearingdatacenter/pages/ download-data-file.

[39] https://www.physionet.org/content/mitdb/1.0.0/. 